\title{
True Ectopia of Right Ovary With Ipsilateral Renal Agenesis
}

\author{
Serbeze Kabashi ${ }^{\mathrm{a}, \mathrm{b}}$, Kreshnike Dedushi ${ }^{\mathrm{a}, \mathrm{b}, \text { d, f, Sefedin Mucaj }}$, c, Naser Ramadani ${ }^{\mathrm{a}, \mathrm{c}}$, \\ Jeton Shatria, c, Astrit Hoxhaj
}

\begin{abstract}
True ectopia of ovarian tissue is a clinical entity which is rarely encountered by the gynecologists. The present report describes a case of true ectopic ovary accompanied with ipsilateral renal agenesis with a review of related literature. A 34-year-old woman was admitted to the department of emergency with abdominal pain localized in the right lower quadrant. The routine tests showed no abnormalities except mild thrombocytosis. The requested abdominal ECHO examination discovered the agenesis of the right kidney joined by consecutive hypertrophy of the left one. And finally, the MRI examination of the abdomen and pelvis confirmed the abovementioned findings and showed another congenital anomaly - the retrocecal position of the right ovary that was consistent with true ovarian ectopic. Association with ipsilateral renal agenesis is indicative of common anomaly originating from urogenital ridge. Physiologic follicles in the right ovary were also noticed as well as two small cysts posterior to ascending colon (separate from ovary). The latest ones were considered as paraovarian cysts or cysts in small surrounding ovarian tissue. We present an extremely rare case of ipsilateral ovarian ectopia and renal agenesis concomitantly. The true ectopic ovary was mimicking some of the symptoms of acute appendicitis. And finally, we provide hints that will help in guiding the management of similar cases.
\end{abstract}

Keywords: Agenesis; Ectopic tissue; Kidney; Ovary

\section{Introduction}

Ectopic ovarian tissue is a rare embryological abnormality

Manuscript accepted for publication April 28, 2016

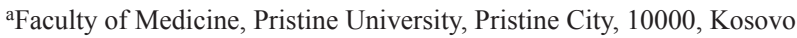
bDepartment of Radiology, Diagnostic Centre, UCCK, Pristine City, 10000, Kosovo

cNational Institute of Public Health of Kosovo, Pristine City, 10000, Kosovo dInternational Health Center "IHC", Pristine City, 10000, Kosovo

'American Hospital, Tirana City, 10004, Albania

${ }_{\mathrm{f}}$ Corresponding Author: Kreshnike Dedushi, Faculty of Medicine, Pristine University; Department of Radiology, Diagnostic Centre, UCCK; International Health Center "IHC", Pristine City, 10000, Kosovo.

Email: kreshnikededushi@gmail.com

doi: http://dx.doi.org/10.14740/jmc2494w with less than 50 cases reported since 1959 [1]. Ectopic ovaries have an estimated prevalence between 1 in 29,000 and 1 in 93,000 gynecologic admissions [2]. A more accurate diagnosis is difficult due to a confusing classification system, as well as their frequently asymptomatic nature. They can be distinguished in supernumerary and accessory ovaries, although this terminology is sometimes confusing [3]. Furthermore, ectopic of the ovary may be rarely accompanied by maldevelopments of the genital tract and changes in the renal system [4]. Appendicitis is the most common general surgical emergency in developed countries. In the majority of female patients, one or more gynecological disorders can mimic acute appendicitis, including pelvic inflammatory disease, ovarian torsion, tubal ectopic pregnancy, hemorrhagic cysts, recent ovulation, tuboovarian masses or infected cysts [5]. We herein describe the clinical findings, diagnostic procedures and management of a 34-year-old female patient with symptoms of acute appendicitis, an ectopic ovary in contact with the appendix, and right (ipsilateral) renal agenesis. It is an extremely rare case of the very few reported in the published work; to the best of our knowledge, it is the second one.

\section{Case Report}

A 34-year-old female patient, gravidity 1, delivery 1, presented with abdominal pain localized in the right lower quadrant and a body temperature of $36.7^{\circ} \mathrm{C}$. Pain was not unbearable and had started 2 months before, but since the localization was in the ileocecal region that usually suggests acute appendicitis, she was brought to the emergency department for further investigation. Pain was not followed by nausea or vomitus or by urinary tract infection. The patient had had regular menstrual cycle every 28 days and had a pregnancy that was completed with a normal birth. She did not have earlier problems in pelvic region and pain in the ileocecal region was not associated with abnormalities in the routine laboratory tests; leucocyte formula and hemogram showed normal values. The exception was mild thrombocytosis, abdominal ultrasound examination was requested and the same showed right renal agenesis and compensatory left kidney hypertrophy. The right ovary was located in retrocecal region and this finding was consistent with true ovarian ectopia. Association with ipsilateral renal agenesis was indicative of common anomaly originating from urogenital ridge. Other findings were physiologic follicles in right ovary and also two small cysts posterior to ascending colon 

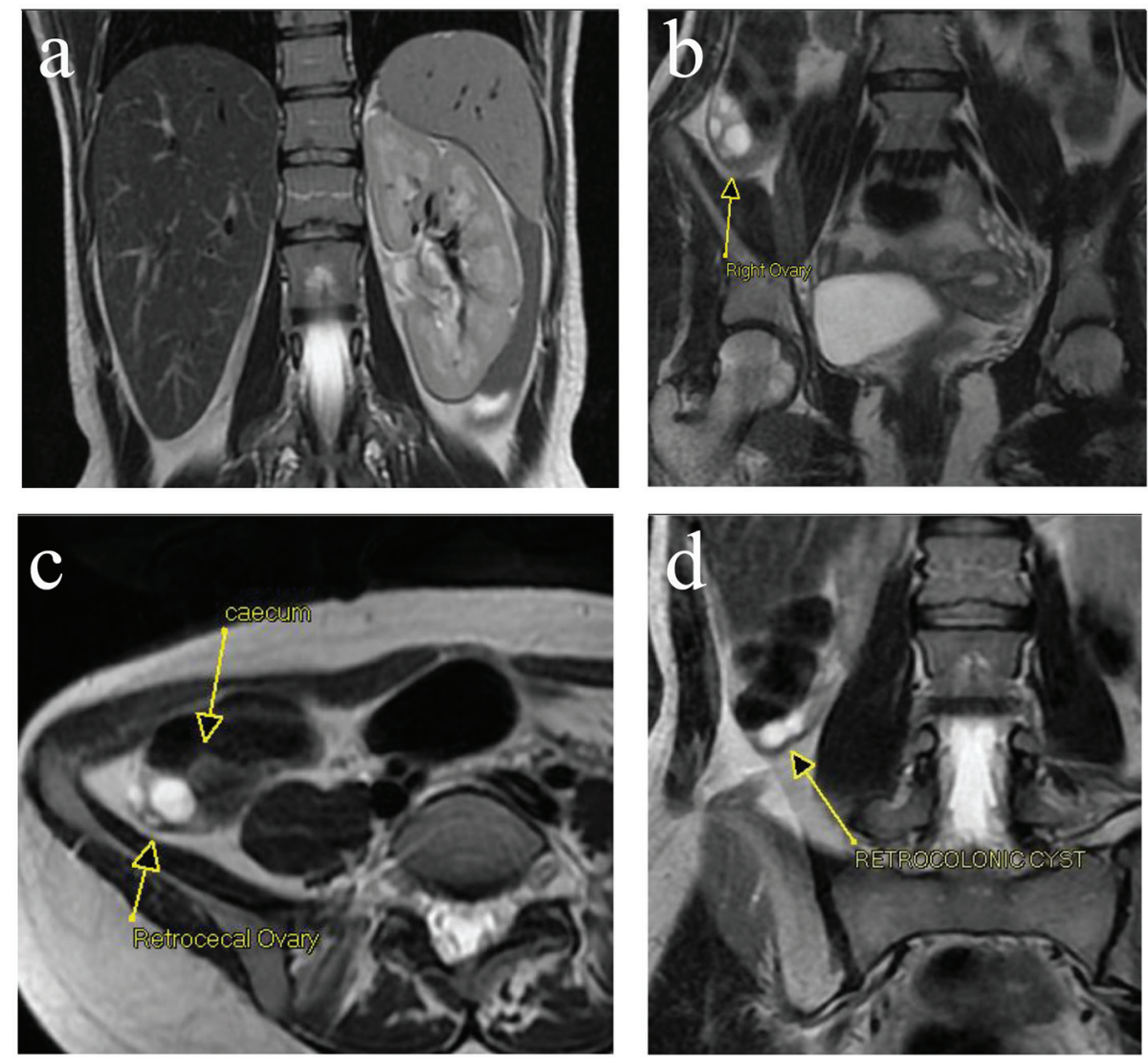

Figure 1. (a) Right renal agenesis and association with ipsilateral renal agenesis is indicative of common anomaly originating from urogenital ridge. (b, c) Right ovary is located in the retrocecal region. It is consistent with true ovarian ectopia. Physiologic follicles in right ovary. (d) Two small cysts posterior to ascending colon (separate from ovary). They were considered as paraovarian cyst or cysts in small surrounding ovarian tissue.

(separate from ovary). They were considered as paraovarian cyst or cysts in small surrounding ovarian tissue (Fig. 1a-d).

\section{Discussion}

Ectopic or undescended ovaries are characterized by their attachment to an area above the level of the common iliac vessels. There are two broadly acknowledged classifications. In 1959, Wharton classified ectopic ovaries as either accessory or supernumerary, distinguished by their relationship to a normal ovary [6]. Accessory ovary was defined as excess ovarian tissue adjacent and connected to a normal ovary. On the other hand, supernumerary ovaries were described as those ovaries situated away from normal ovaries (e.g. in the retroperitoneum or omentum). This classification implies the presence of two normal ovaries and also does not account for abnormally located ovarian tissue secondary to previous pelvic surgery, such as found in ovarian implant/remnant syndrome. On the other hand, the review by Lachman and Berman found that almost $50 \%$ of the reported cases since 1959 were in patients with previous pelvic surgery. They also proposed a new classification of abnormally located ovarian tissue to further clarify the issue. They suggested that ectopic ovary should be the proper term and that this can be further sub-classified as: 1) post-surgical implant; 2) post-inflammatory implant; and 3) true (ectopic) ovarian tissue. Both the genital and the urinary system develop from the intermediate mesoderm, which extends along the dorsal wall of the embryo. This is the primordium of the urogenital ridge which can be divided into two parts: the nephrogenic ridge which gives rise to the urinary system and the gonadal ridge which becomes the genital system. The primitive genital system in the two sexes is similar. The gonads are derived from three sources: mesothelium, mesenchyme and primordial germ cells. By 18 weeks, many primordial ovarian follicles have already formed. The ovaries, located on the dorsal wall of the embryo, begin their descent by 20 weeks. Guided by the gubernaculum and processus vaginalis, the ovaries descend to a point inferior to the pelvic brim. The gubernaculum gives rise to the ovarian ligaments and round ligaments of the uterus. Ectopia of the ovary may be accompanied by additional maldevelopments of the genital tract which sometimes are accompanied by anomalous changes in the renal system. Renal agenesis is a relatively common congenital anomaly, although its etiology is unknown. This kind of agenesis may be unilateral or bilateral and is generally thought to result from: 1) absence of the metanephric blastema; 2) ureteral bud maldevelopment; or 3) lack of induction of the metanephric blastema by the ureteral bud. 
Unilateral renal agenesis is usually an incidental finding. Renal agenesis is also associated with ipsilateral urogenital anomalies [7]. The ovary, which forms from the urogenital ridge, is present on the affected side but may be higher in the abdomen than its normal pelvic location [8]. The ovaries in such patients may be found in the upper abdomen, at the level of the pelvic brim, or in the inguinal canal. In our case, the right ovary was located in the right fossa and the right kidney was absent. It is necessary to mention that renal agenesis and true ectopia of the ovary are both anomalies of the urogenital ridge, the common mesodermal ridge that forms the developing gonad and the mesonephros. Renal agenesis occurs when the nephrons fail to develop and the ureteric bud fails to branch. It may also arise if the interaction between the metanephric mesoderm and the ureteric bud fails to occur. The descent of the ovary into the pelvis depends on the formation of the cranial genital ligament, which forms the suspensory ligament of the ovary, and the caudal genital ligament, which forms the proper ligament of the ovary and the round ligament of the uterus [9].

\section{Conclusion}

According to the facts mentioned above, we assume that in our case report the patient suffered a defect of the urogenital ridge which led to two correlated but independent abnormalities in two different steps of the development of the urogenital system. The uterus was normal because the paramesonephric ducts fused and the ovary failed to descend due to a malformation of its ligaments. We also characterize the ectopic ovarian tissue of our case as a true ectopic ovary, undescended due to a combined malformation of the urogenital tract which also appeared with the unilateral renal agenesis. In our case, we assumed that the ectopic ovarian tissue was the cause of the abdominal pain that was not very typical for appendicitis and was not joined by high temperature. The disturbing pain occurred usually during premenstrual time. After employment of different imaging examinations, we have verified that the cause of disturbances was the ectopic ovary. The clinicians need to be careful during differentiation of origins of the pain that comes from the ileocecal region. The abdominal surgeons are usually not informed about this very rare anomaly and enter the surgery presumably for acute appendicitis or for more frequent acute gynecological problems that can mimic it (parafollicular cysts near the ovary can grow and burst and this will result with fluid in Douglas).

Magnetic resonance imaging (MRI) is an ideal option for the diagnosis and management of an ectopic ovary. MRI is a non-invasive, highly sensitive and specific radiologic modality in the diagnosis of genital tract and concurrent renal anomalies and follow-up in patients with infertility or recurrent pelvic pain. In the presented case, it confirmed the absence of the right kidney and the presence of the ectopic ovary in the right ileocecal fossa. The renal function was in normal range and we thought there was no need for further renal agenesis evaluation by IV pyelogram. In conclusion, we present an extremely rare case of ipsilateral ovarian ectopia and renal agenesis concomitantly. The true ectopic ovary was also mimicking some of the symptoms of acute appendicitis. We finally provide hints that will help to guide the management of similar cases.

\section{Conflicts of Interest}

The authors have no conflicts of interest to declare.

\section{Author Contributions}

All authors of this paper have contributed in all phases. Final proof reading was made by the first author. All authors have read and approved the final manuscript.

\section{References}

1. Bayramov V, Sukur YE, Cetinkaya E, Berker B. Ectopic ovary autotransplanted over rectosigmoid colon: a case report. Fertil Steril. 2009;92(4):1496 e1415-1496.

2. Watkins BP, Kothari SN. True ectopic ovary: a case and review. Arch Gynecol Obstet. 2004;269(2):145-146.

3. Lachman MF, Berman MM. The ectopic ovary. A case report and review of the literature. Arch Pathol Lab Med. 1991;115(3):233-235.

4. Uyar I, Gulhan I, Sipahi M, Hanhan HM, Ozeren M. Ectopic ovary confirmed by ovarian stimulation in a case of unicornuate uterus. Fertil Steril. 2011;96(2):e122-124.

5. Shin LK, Jeffrey RB. Sonography and computed tomography of the mimics of appendicitis. Ultrasound Q. 2010;26(4):201-210.

6. Wharton LR. Two cases of supernumerary ovary and one of accessory ovary, with an analysis of previously reported cases. Am J Obstet Gynecol. 1959;78:1101-1119.

7. Mishra A. Renal agenesis: report of an interesting case. Br J Radiol. 2007;80(956):e167-169.

8. Reichman DE, Laufer MR. Congenital uterine anomalies affecting reproduction. Best Pract Res Clin Obstet Gynaecol. 2010;24(2):193-208.

9. Litos MG, Furara S, Chin K. Supernumerary ovary: A case report and literature review. J Obstet Gynaecol. 2003;23(3):325-327. 